\title{
ÉVALUATION DU RISQUE DANS LA MALADIE DU SOMMEIL : APPORT DE LA MODÉLSATION MATHÉMATIQUE
}

GOUTEUX J.P.*

Summary: RISK EVALUATION IN SLEEPING SICKNESS: A MATHEMATICAL CONTRIBUTION

The concept of "risk" is important in epidemiology but is often used in a confused way for sleeping sickness. Using a rigorous approach resulting from mathematical modelling, new virtual entomological risk indicators and parasitological transmission indexes derived from the basic reproduction rate $R_{0}$ are proposed and discussed.

KEY WORDS : trypanosomosis, risk, Glossina, tsetse, entomological index, parasitology, epidemiology, mathematical modelling.

\footnotetext{
I
} 'Organisation mondiale de la santé estime qu'en Afrique 60 millions de personnes sont soumises au risque de trypanosomose humaine africaine (THA) ou maladie du sommeil (Cattand, 2001). Ce risque, en Afrique de l'Ouest et en Afrique Centrale, est lié à la fois à la présence de malades qui constituent le principal réservoir de parasites, et au contact avec les glossines ou mouches tsé-tsé (Diptères, Glossinidés) qui sont les seuls vecteurs connus de cette maladie. Le risque épidémiologique peut aussi bien désigner un risque entomo-parasitologique, ou risque réel de transmission, qu'un simple "indice entomologique", c'est-à-dire un risque virtuel (ou potentiel) lié à l'exposition aux populations de tsé-tsé estimée à partir d'une connaissance plus ou moins fine de leurs caractéristiques. Il peut s'agir aussi d'un risque attribuable à une population humaine particulière dans des circonstances données (Wyatt et al., 1985; Méda et al., 1993; Okia et al., 1994; Grébaut et al., 2001). Ces risques catégoriels ou "attributable risks" (Last, 1983) dépendent de l'exposition aux vecteurs de groupes de la population (liée à leur comportement), et/ou de susceptibilités différentes (liées à l'état sanitaire, l'hérédité ou encore l'immunité). Ils intègrent implicitement l'aspect entomologique, mais sans connaître les particularités des populations de vecteurs impliqués. Ce type

\footnotetext{
* IRD (UR GEODES), Laboratoire MAT (Université de Yaoundé) I, BP 1857, Yaoundé, Cameroun.

Correspondance : Jean-Paul Gouteux.

IRD, B.P. 1857, Yaoundé, Cameroun.

Tél.: + 2372201508 - Fax: + 2372201854 .

E-mail : jean-paul.gouteux@ird.fr
}

\begin{abstract}
Résumé :
Le concept de "risque" est important en épidémiologie, mais il est souvent utilisé de manière confuse pour la maladie du sommeil. Une approche rigoureuse issue de la modélisation mathématique est utilisée pour proposer et discuter de nouveaux indicateurs du risque entomologique virtuel et du risque parasitologique réel, dérivés du taux de reproduction $R_{0}$.
\end{abstract}

MOTS CLÉS : trypanosomose, risque, glossine, tsé-tsé, indice entomologique, parasitologie, épidémiologie, modèles mathématiques.

de risque catégoriel n'est pas pris en compte ici, pas plus que les approches perceptives du risque (psychologiques et sociologiques) ou que les approches économiques et politiques (voir Coppieters et al., 2004 pour une définition de ces différentes approches).

\section{L'ÉVALUATION DU RISQUE RÉEL DE TRANSMISSION}

Pour qu'il y ait transmission, il faut que le parasite soit disponible et donc présent dans un réservoir animal ou humain. Smith et Rennison (1958) définissent le "challenge", comme étant le nombre moyen de piqûres infectantes qu'un hôte reçoit par unité de temps. C'est évidemment une définition idéale, mais pratiquement et éthiquement inconnaissable pour la THA. Il faudrait en effet faire des captures sur appât humain dans une zone de transmission, ce qui n'est pas acceptable. En 1964, Cawdery et Simmons (in Rogers, 1979, 1980) proposent un indice pour évaluer ce "challenge" qui est le simple produit des densités apparentes et du taux d'infection moyen. Wilson, Dar et Paris (1972) proposent un autre "index of challenge", le produit du taux d'infection des mouches par la probabilité qu'une piqûre infectante contamine un humain. Ces indices entomo-parasitologiques font intervenir la circulation du parasite à travers sa présence dans le vecteur. Ils nécessitent de disposer d'un recueil très complet des données entomologiques, parasitologiques et médicales sur le terrain.

\section{L'ÉVALUATION ENTOMOLOGIQUE DU RISQUE}

Contrairement au "challenge" mentionné plus haut, les indices d'évaluation entomologique mesurent le risque 
créé par une population de vecteurs en cas d'importation du parasite. Ce risque est donc virtuel et non un réel danger de contamination. Rogers (1979) s'est interrogé sur ce qui, des densités apparentes ou du comportement alimentaire des glossines, serait le facteur principal de la transmission, sans apporter de réponse. Plus tard, ce même auteur (Rogers 1980, 1985) a estimé qu'il y avait une relation simple et linéaire entre les densités apparentes du vecteur et les prévalences des trypanosomoses animales et humaines. Ce qui peut être vrai dans le cas des infections du bétail ne l'est pas dans le cas des infections humaines (Opiyo et al., 1982). En effet, Nash (1944) et Morris (1952) avaient observé que l'intensité de la transmission pouvait être en opposition avec la densité du vecteur. Le constat qu'il existe partout des zones à très forte densité glossinienne sans maladie du sommeil est trivial. Il s'agit d'un phénomène comparable à celui de l'absence de paludisme dans certaines zones où les anophèles sont présents en nombre. L'indice de risque proposé par Laveissière et al. (1986, 1994, 2000) est une tentative plus fine pour identifier le risque lié à une population particulière de vecteurs, définie par son biotope où a été effectué le piégeage. Cet indice prend en compte une estimation des nombres de glossines ténérales (voir plus bas) susceptibles de s'infecter et de glossines gorgées sur humain, ainsi que le taux de survie. Cependant, vouloir attribuer un risque selon les biotopes est en contradiction avec la mobilité du vecteur. En effet, les glossines qui se reposent s'accouplent, se nourrissent dans ces différents biotopes appartiennent à une même population. De plus, cet indice utilise un "nombre estimé" de glossines se nourrissant sur humains dans un biotope donné. L'idée étant qu'un faible taux de repas de sang sur humains pourra être compensé par une forte densité à cet emplacement. En réalité, c'est la probabilité pour une glossine de piquer un humain (nombre sans dimension) qui a une signification épidémiologique, car elle intègre la dilution des hôtes humains par rapport à la totalité des hôtes accessibles. Elle donne une information pertinente sur le nombre d'humains et d'animaux disponibles comme hôtes. L'ignorer revient à perdre une information essentielle. La modélisation mathématique, en permettant une approche rigoureuse, est particulièrement utile pour la compréhension et la quantification des processus épidémiologiques. Nous l'utilisons ici dans une optique déterministe pour proposer de nouveaux indicateurs entomologique et entomoparasitologique.

\section{LE PROCESSUS ÉPIDÉMIOLOGIQUE}

Trypanosoma brucei gambiense, agent de la maladie du sommeil, est transmis à un humain sain par une glossine infectée. Après une période d'incubation variable, d'une douzaine de jours en moyenne (Rogers, 1988a et b), le malade rentre en première phase (ou phase lymphatico-sanguine) et devient infectant pour les glossines qui viennent se gorger sur lui. Ce sont les jeunes glossines, dites ténérales, qui ont la plus forte probabilité de s'infecter. Les glossines ténérales (du latin tener $=$ mou, par référence à leur cuticule encore tendre) sont âgées de moins de trois jours et n'ont pas encore pris de repas de sang. L'infection ultérieure est possible (Gooding, 1988) notamment après un jeûne prolongé (Gingrich et al., 1982b) mais reste relativement négligeable (Harley, 1971; Lambrecht, 1980; Gingrich et al., 1982a). La forme gambienne de la maladie est d'évolution lente. En première phase, le malade présente peu de troubles et pourra, en continuant à vaquer à ses activités, contaminer un grand nombre de tsé-tsé. Finalement, le parasite envahit le système nerveux central, déterminant la seconde phase (ou phase méningo-encéphalitique). Le malade est alors devenu très peu infectant pour les tsé-tsé et s'expose moins aux piqûres. Il est plus difficile à guérir et condamné à une issue fatale s'il n'est pas soigné. Le traitement le plus répandu est toujours à base de dérivés arsenicaux dangereux et peut entraîner jusqu'à $7 \%$ de mortalité. Certains animaux sauvages et/ou domestiques peuvent constituer des réservoirs secondaires et supplémentaires pour Trypanosoma brucei gambiense (Simo et al., 2000; Nkinin et al., 2002).

\section{PARAMĖTRES INTERVENANT DANS LA TRANSMISSION}

(tableau I)

$\tau$ a symbolique utilisée ici est reprise des modèles mathématiques d'Artzouni et Gouteux (1996a et b). Jn considère comme "unité épidémiologique", la zone formée par un village, un campement ou un ensemble de campements proches et leur environnement, associé à l'activité humaine (terroir villageois, zone forestière, plantations, champs et friches environnants). Les populations humaines de $H$ individus y sont confrontées à une population fluctuante de $V(\mathrm{t})$ glossines, dont le nombre à l'équilibre est $V$. C'est ce paramètre qui est utilisé ici, bien qu'en réalité les populations animales ne soient pas stables, mais fluctuent autour d'une densité d'équilibre. Cependant Jarry et al. (1996) ont montré que les populations de tsétsé restent assez proches de l'équilibre. Le rapport $\mathrm{V} / \mathrm{H}$ des effectifs des humains et des tsé-tsé représente la "densité vectorielle" exprimée en nombre de tsé-tsé par habitant. Les tsé-tsé forment une population ouverte, c'est-à-dire non isolée, avec des flux d'émigration et d'immigration journalières importants. Pour les humains, 


\begin{tabular}{llll}
\hline & Population humaine (fermée) & Population de glossines (ouverte) \\
\hline$H$ & $\begin{array}{l}\text { Nombre d'individus de l'unité épidémiologique } \\
\text { (village ou campements) }\end{array}$ & $\mathrm{V}$ & $\begin{array}{l}\text { Ensemble des tsé-tsé ayant une probabilité non } \\
\text { négligeable d'entrer en contact avec un individu } \\
\text { de } H\end{array}$ \\
$\mathrm{Ha}$ & Malades en première phase & $\mathrm{Va}$ & Tsé-tsé infectantes \\
$\mathrm{Hr}$ & Malades en seconde phase & $\mathrm{m}$ & Taux de mortalité \\
$(\mathrm{Ha}+\mathrm{Hr}) / H$ & Prévalence & $\mathrm{b}$ & Taux de natalité \\
$r_{1}$ & Taux de passage de la première à la seconde & $\mathrm{Vb}$ & Nombre de ténérales \\
& phase & $1 / q$ & Durée de la période d'incubation \\
$1 / r_{1}$ & Durée de la première phase & $1 / \mathrm{m}$ & Durée moyenne de vie \\
$\tau_{3}$ & Probabilité de développer l'infection & $\tau_{1}$ & Taux de repas de sang sur humain \\
& & $\tau_{2}$ & Probabilité de développer l'infection \\
\hline
\end{tabular}

Tableau I - Principaux paramètres intervenant dans la transmission.

on considère un village stable où ces flux sont négligeables aux mêmes échelles de temps.

\section{POPULATIONS HUMAINES}

La population $H$ comprend les sous-populations de $H_{a}$ malades en première phase et de $H_{r}$ malades en deuxième phase. La prévalence de la maladie est $\left(H_{a}+H_{r}\right) / H$ et le nombre d'individus sains dans la population est donc $H-\left(H_{a}+H_{r}\right)$. La probabilité qu'une piqûre infectante contamine un hôte vertébré sain est $\tau_{3}$. Cet indice, nommé "transmission index" par Wilson, Dar et Paris (1972), est estimé à 0,62. La virulence moyenne de la souche de T. b. gambiense pour les populations humaines concernées est exprimée par le taux de passage $r_{1}$ des malades en première phase à la deuxième phase. Ainsi $1 / r_{1}$ est la durée moyenne de la première phase paucisymptomatique, pendant laquelle les individus peuvent diffuser le parasite, d'où l'importance de ce paramètre estimé avant traitement. Chez certains malades, ce taux $r_{1}$ pourrait être extrêmement petit, déterminant une forme chronique de la maladie, ceuxci joueraient alors le rôle de réservoir cryptique de parasite. La durée de la phase d'incubation étant relativement courte ( 7 à 12 jours) elle n'est pas prise ici en considération.

\section{POPULATION DE VECTEURS}

Pour une population $V$ et un pas de temps $t$, les taux de mortalité $(m)$ et de natalité $(b)$ sont supposés constants à l'équilibre. La durée de vie moyenne dans l'environnement étudié est $1 / m$ (exprimée dans le pas de temps considéré). Elle est en général de 30 à 45 jours. Le nombre de mouches ténérales est $V b$ (si $b$ est le rapport net des naissances sur la mortalité par période de trois jours). La population $V$ comprend $V_{a}$ tsé-tsé infectantes, vecteurs de la maladie et $V_{i}$ tsé-tsé incubantes, porteur de formes immatures du parasite, non infectantes pour les hôtes vertébrés. Le taux de repas de sang sur homme $\tau_{1}$ est la probabilité de piquer un humain pour cette population glossinienne. La capacité vectorielle intrinsèque (Le Ray, 1989) $\tau_{2}$ ou "compétence vectorielle" est la probabilité pour qu'une mouche ténérale piquant un malade en première période devienne infectante, c'est-à-dire qu'elle développe une infection mature. Ce paramètre $\tau_{2}$ dépend de l'espèce, voire de la population de tsé-tsé concernée et du stock de trypanosomes circulant. $q$ est le taux de sortie de la phase d'incubation du parasite $V_{i}$ à la phase d'infection active $V_{a}$. La durée de la phase d'incubation $(1 / q)$ est de 15 à 25 jours. Elle est relativement longue par rapport à la moyenne de vie des tsé-tsé.

\section{APPROCHE MATHÉMATIQUE}

\section{LE TAUX DE REPRODUCTION $R_{O}$}

Les modèles mathématiques déterministes de la transmission d'une maladie vectorielle ont introduit $R_{0}$, taux de reproduction. C'est le nombre moyen d'humains qui deviendront éventuellement infectés via les tsé-tsé à partir d'un seul malade introduit dans une population saine. Ainsi, si $R_{0}>1$, l'épidémie s'étend, si $R_{0}<1$, elle évolue vers l'extinction. Ce taux s'exprime lorsque la population de tsé-tsé a atteint son équilibre $V$ et en l'absence d'immigration de mouches infectées. Introduit par Kermack et McKendrick (Anderson et al., 1982), ce taux $R_{O}$ est fondamental en modélisation épidémiologique. Certains auteurs, à la suite de Dietz (1988), notamment Rogers (1988b), Artzrouni et Gouteux (1999) l'ont décomposé en un produit $R_{1} R_{2}$ pour le rendre plus explicite (notons qu'il s'agit dans tous les cas de nombre moyen d'humains ou de tsé-tsé).

Le taux $R_{O}=R_{1} R_{2}$ est donné par la formule suivante :

$$
R_{0}=\frac{V b q \tau_{1}^{2} \tau_{2} \tau_{3}}{H r_{1}(q+m) m}
$$

Si $R_{1}$ est le nombre de vecteurs infectés par ce "patient source", on aura :

$$
R_{1}=\frac{V b \tau_{1} \tau_{2}}{r_{1} H}
$$


En effet, chacune des $V b$ tsé-tsé ténérales a une probabilité $\tau_{1} / H$ de piquer ce patient et $\tau_{1} \tau_{2} / H$ s'être infectée. Ce patient aura une "carrière infectieuse" de durée moyenne $1 / r_{1}$ qui est la durée de la phase infectante et qui lui aura permis d'infecter $R_{1}$ tsé-tsé. Chacune des $R_{1}$ tsé-tsé infectées infectera à son tour un nombre d'humains égal à $R_{2}$ qui s'écrit :

$$
R_{2}=\frac{q \tau_{1} \tau_{3}}{m(q+m)}
$$

En effet, chaque tsé-tsé ayant piqué un malade aura une probabilité $q /(q+m)$ d'atteindre le stade infectant, puisque $q$ est le taux de passage du stade de l'incubation à celui de l'infection active et $m$ le taux de mortalité. Sa "carrière infectieuse" sera $1 / m$ qui est l'espérance de vie des tsé-tsé à l'émergence ou durée moyenne de vie. Comme $\tau_{1}$ est la probabilité de piquer un humain et $\tau_{3}$ la probabilité pour que l'infection se développe chez cet humain, elle contaminera donc $R_{2}$ humains. Le taux de reproduction $R_{O}=R_{1} R_{2}$ est le produit du nombre moyen de vecteurs infectés par le nombre moyen de nouvelles infections humaines générées par chacune des nouvelles tsé-tsé infectées. On voit donc que le dépistage des malades qui diminue $1 / r_{1}$, la durée de la carrière infectieuse des malades, agit sur $R_{1}$. La lutte antivectorielle qui augmente le taux de mortalité $m$ du vecteur, agit quant à elle sur $R_{2}$.

\section{LA VITESSE DE CONTAMINATION $M_{O}$}

On peut en simplifiant $R_{0}$ obtenir la vitesse de contamination $M_{O}$ qui est le nombre moyen de nouvelles infections humaines par unité de temps.

$$
M_{O}=\frac{V b q \tau_{1}^{2} \tau_{2} \tau_{3}}{H m(q+m)}
$$

\section{LE POTENTIEL DE DIFFUSION $Q_{O}$}

Le paramètre $Q_{0}$ mesure le potentiel de diffusion de l'épidémie ou taux de contamination intrinsèque. Il est proportionnel à $M_{O}$ pour $m, b$ et $q$ fixés.

$$
Q_{0}=\frac{V}{H} \tau_{1}^{2} \tau_{2} \tau_{3} 10^{4}
$$

Avec $\tau_{1}$ exprimé en pourcentage. Notons que les paramètres $\tau_{2}$ et $\tau_{3}$, probabilités que l'infection se développe chez les vecteurs et chez les humains sont difficilement estimables. Il s'agit des "terrains" humains et glossiniens qui peuvent être très variables. Par exemple, une population d'allogènes burkinabé pourra être beaucoup plus sensible ( $\tau_{3}$ plus grand) que les autochtones ivoiriens d'un foyer forestier. De même $\tau_{2}$ varie selon les espèces et les stocks de $T$. $b$. gambiense. $Q_{O}$ est à rapprocher de l'indice de contamination nouvelle (ICN), rapport du nombre de nouveaux malades pendant une période donnée sur la population totale examinée $H_{a}(\Delta \mathrm{t}) / H$, taux d'incidence classique utilisée en épidémiologie de la maladie du sommeil. En admettant que $Q_{o}=H_{a}(\Delta \mathrm{t}) / H$ à un facteur de proportionnalité près, on voit que le produit $V \tau_{1}^{2}$ est proportionnel au nombre de nouveaux cas.

Nous proposons l'indice $Q_{o}$, potentiel de diffusion de l'épidémie, comme nouvel indice de risque entomologique virtuel pour une population humaine donnée $H$, (appartenant à une unité de transmission donnée), confrontée à une population de vecteur donnée $V$ ayant une probabilité moyenne $\tau_{1}$ de prendre un repas de sang sur humain.

\section{RISQUE FAISANT INTERVENIR LA DIFFUSION DU PARASITE}

Le contact des humains avec les tsé-tsé ne présente un risque que si la population de tsé-tsé est susceptible d'avoir été elle-même au contact de porteurs de l'élément infectieux. Pour évaluer le risque réel de transmission dans différents foyers et micro foyers d'une vaste zone d'endémie, il est judicieux de faire intervenir le taux de prévalence de la maladie $H_{a} / H$ et le taux d'infection des vecteurs $V_{a} / V$. Le risque d'infection d'une tsé-tsé est proportionnel au produit de $\tau_{1}$ $H_{a} / H$ par $\tau_{2}$.

$$
R_{V}=\frac{H_{a}}{H} \tau_{1} \tau_{2} 10^{4}
$$

Le risque de contamination humaine est proportionnel au produit $\tau_{1} V_{a} / V$ par $\tau_{3}$.

$$
R_{H}=\frac{V_{a}}{V} \tau_{1} \tau_{3} 10^{4}
$$

Le facteur $10^{4}$ indique que ces taux sont exprimés en pourcentage pour être plus maniables. Usuellement $\tau_{2}$ est estimé à 0,1 et $\tau_{3}$ à 0,62 . Si le seul réservoir de parasites est humain, $R_{V}$ est alors un bon indice de la circulation des tsé-tsé infectées. Par exemple, s'il n'y a pas de malades dépistés, $R_{V}=0$, et si, simultanément, $R_{H}$ est non nul, cela indique soit que le dépistage des malades n'a pas été exhaustif, soit qu'il existe pour les tsé-tsé une source animale de contamination par Trypanosoma brucei gambiense. Une étude précise de ces deux indices dans un foyer donné devrait permettre de répondre à la question si controversée de l'existence, ou non, d'un réservoir animal de parasite dans ce foyer. Ces nouveaux indices permettent d'évaluer le risque auquel était soumise la population dans les lieux de contamination probable au moment du dépistage des malades, estimé par une enquête approfondie, car les malades dépistés dans un espace particulier peuvent ne pas avoir été contaminés dans ce même espace. Dans une situation d'urgence sanitaire, ces deux indices peuvent permettre d'établir une hiérarchie des foyers selon leur dangerosité. Des mesures immédiates de 
lutte antivectorielle peuvent alors être prises en conséquence.

\section{RISQUE D'ERREUR SUR LE RISQUE}

Tous ces indices d'évaluation d'un risque de transmission utilisent des taux basés sur une approximation de la réalité à partir d'échantillons. Il est bien sûr nécessaire de prendre en compte le risque d'erreur statistique sur $\tau_{1}, V_{d} / V, H_{d} / H$ et de calculer l'intervalle de confiance à $5 \%$ de ce risque selon les méthodes habituelles. Cette erreur statistique, liée à la caractéristique de l'échantillon, peut seule permettre de savoir s'il y a ou non des différences entre les risques calculés pour différents foyers. Le modèle utilisé doit être adapté aux caractéristiques de l'échantillon : gaussien (distribution normale) ou autre (distribution de Poisson ou distribution binominale négative), souvent mieux adaptées aux spécificités de la THA.

\section{DISCUSSION}

L a nature d'événements rares des processus épidémiologiques concernant la trypanosomose humaine rend tout particulièrement difficile l'évaluation du risque. $Q_{O}$ est une évaluation dérivée de $R_{O}$ basée sur les modèles mathématiques classiques. Il s'applique à une population humaine très localisée (le village ou le campement). Mathématiquement, $Q_{o}$ indique le risque de propagation de la maladie après l'introduction d'un malade en première phase ou d'une tsé-tsé infectante, qui constituent les deux "sources" initiales possibles de parasites dans l'espace de l'unité épidémiologique. Sa formule rend-t-elle compte du risque associé à de grosses agglomérations humaines? Les gros villages entraînent une anthropisation forte du milieu qui diminue de façon drastique les points de contact (Gouteux et al., 1993a et b). Il est donc possible qu'une forte densité humaine augmente de façon non linéaire l'effet de dilution lié à $1 / H$.

Tout indice basé uniquement sur des paramètres entomologiques et non parasitiologiques n'indique qu'un risque virtuel de développement de l'épidémie dans la zone étudiée et non un risque réel de contamination. C'est-à-dire qu'à un niveau de risque virtuel très élevé peut correspondre un risque réel nul, s'il n'y a pas de parasite en circulation. Pour certains auteurs, ce type d'indice ne serait pas réfutable expérimentalement (falsifiable au sens de Popper, 1985). Ainsi les discordances observées entre les valeurs de l'indice de Laveissière et al. (1986, 1994, 2000) et les observations épidémiologiques faites dans différents foyers ivoiriens (Fournet et al., 2001) sont expliquées par la nature virtuelle du risque (Fournet et al., 2001). Il est clair que si les coïncidences sont établies comme preuves et les discordances éliminées comme non pertinentes par définition, il n'y a pas de possibilité de réfutation. Cependant, même s'ils indiquent un risque potentiel, ces indices restent falsifiables au sens de Popper. Il est en effet possible de les discriminer en les comparant aux taux de prévalence. Il serait donc intéressant de relier de façon systématique les indices entomologiques avec l'incidence ou la prévalence dans les différents foyers actifs où des études entomologiques approfondies ont été effectuées. Des corrélations avec les observations médicales pourront être alors faites pour vérifier quel indice virtuel est le plus pertinent.

\section{REMERCIEMENTS}

L'auteur remercie Régis Pouillot pour sa relecture attentive du manuscrit.

\section{RÉFÉRENCES}

ANDERSON R.M. \& MAY R.M. The population biology of infectious diseases. Springer Verlag, Berlin, 1982, 368 p.

Artzrouni M. \& Gouteux J.P. A compartmental model of vector-borne diseases: application to sleeping sickness in central Africa. Journal of Biological Systems, 1996a, 4, 459-477.

Artzrouni M. \& Gouteux J.P. Control strategies for sleeping sickness in Central Africa: a modelbased approach. Tropical Medicine and International Health, 1996b, 1, 753764.

Artzrouni M. \& Gouteux J.P. A Model for the spread of sleeping sickness, in: Applied mathematical modeling: a multidisciplinary approach. Shier D.R. \& Wallenius K.T. (eds), CRC Press, Boca Raton, 1999, pp. 71-92.

CatTand P. Donnez moi deux bombardiers ou cinq hélicoptères. Médecine Tropicale, 2001, 61, 311-312.

Coppieters Y., Parent F., Lagasse R. \& PietTe D. Évaluation des risques, une approche pluridisciplinaire en santé publique. Environnement, Risques E Santé, 2004, 3, 45-52.

DiETZ K. Density dependence in parasite transmission dynamics. Parasitolology Today, 1988, 4, 91-97.

Fournet F., Koné A., Méda A.H., Traoré S. \& Hervouët J.P. Intégration des facteurs démographiques à la caractérisation d'un espace à risque de maladie du sommeil en Côte d'Ivoire. Médecine Tropicale, 2001, 61, 372-375.

Gingrich J.B., Ward R.A., Macken L.M. \& Esser K.M. African sleeping sickness: new evidence that mature tsetse flies (Glossina morsitans) can become potent vectors. Transactions of the Royal Society of Tropical Medicine and Hygiene, 1982a, 76, 479-481.

Gingrich J.B., Ward R.A., Macken L.M. \& Schoenblechler M.J. Trypanosoma brucei rhodesiense (Trypanosomatidae): factors influencing infection rates of a recent human isolate in the tsetse Glossina morsitans (Diptera: Glossinidae). Journal of Medical Entomolology, 1982b, 9, 268-274. 
Gooding R.H. Infection of post-teneral tsetse fly (Glossina morsitans morsitans and Glossina morsitans centralis) with Trypanosoma brucei brucei. Canadian Journal of Zoology, 1988, 66, 1289-1292.

Gouteux J.P., Kounda-Gboumbi J.C., D'Amico F., Wagner C., Noutoua L. \& Bailly C. Enquête épidémiologique pour la recherche des lieux de contamination probables dans un foyer centrafricain de maladie du sommeil. Bulletin de l'Organisation Mondiale de la Santé, 1993a, 71, 605-614.

Gouteux J.P., Kounda-Gboumbi J.C., Noutoua L., D’Amico F., Bailly C. \& Roungou J.B. Man-fly contact in the Gambian trypanosomiasis focus of Nola-Bilolo (Central African Republic). Tropical Medicine and Parasitology, 1993b, 44, 213218.

Grébaut P., Bodo J.M., Assona A. et al. Recherche des facteurs de risque de la trypanosomose humaine africaine dans le foyer de Bipindi au Cameroun. Medecine Tropicale, 2001, 61, 377-383.

Harley J.W. The influence of the age of the fly at the time of the infecting feed on infection of Glossina fuscipes with Trypanosoma rhodesiense. Annals of Tropical Medicine and Parasitology, 1971, 65, 191-196.

Jarry M., Gouteux J.P. \& Khaladi M. Are tsetse fly populations close to equilibrium? Acta Biotheoritica, 1996, 44, 317-333.

LAMBRECHT F.L. Ecological and physiological factors in the cyclic transmission of African trypanosomiasis. Insect Science and its Applications, 1980, 1, 47-54.

LAST J. A dictionary of epidemiology. Oxford University Press, Oxford, 1983, 141 p.

Laveissière C., Couret D. \& Hervouët J.P. Localisation et fréquence du contact homme/glossine en secteur forestier de Côte d'Ivoire. I. Recherche des points épidémiologiquement dangereux dans l'environnement végétal. Cahiers ORSTOM, série Entomologie médicale et Parasitologie, 1986, 24, 21-35.

LAveissière C., SANÉ B. \& MÉDA H.A. Measurement of risk in endemic areas of human African trypanosomiasis in Côte d'Ivoire. Transactions of the Royal Society of Tropical Medicine and Hygiene, 1994, 88, 645-648.

Laveissière C., Grébaut P., Herder S. \& Penchenier L. Le risque de transmission, in : Les glossines vectrices de la trypanosomiase humaine africaine. IRD, Paris, 2000, pp. 121123.

LE RAY D. Vectors susceptibility to African trypanosomes. Annales de la Société belge de Médecine tropicale, 1989, 69 (suppl. 1), 165-171.

Méda H., Laveissière C., De Muynck A., Doua F. \& Diallo P.B. Les facteurs de risque de la trypanosomiase humaine africaine dans les foyer endémique de Côte d'Ivoire. Médecine Tropicale, 1993, 53, 83-92.

Morris K.R.S. The ecology of epidemic sleeping sickness. 1. The significance of location, Bulletin of Entomological Research, 1952, 42, 427-433.

NASH T.A.M. A low density of tsetse flies associated with a high incidence of sleeping sickness. Bulletin of Entomological Research, 1944, 35, 51.

Nkinin S.W., Njiokou F., Penchenier L., Grebaut P., Simo G. \& HeRder S. Characterization of Trypanosoma brucei s.l. subspecies by isoenzymes in domestic pigs from the Fontem sleeping sickness focus of Cameroon. Acta Tropica, 2002, 81, 225-232.

Okia M., Mbulamberi D.B. \& De Muynck A. Risk factors assessment for T. $b$. rhodesiense sleeping sickness acquisition in S.E. Uganda. A case-control study. Annales de la Société belge de Médecine tropicale, 1994, 74, 105-112.

Opiyo E.A., Alushula H., Kuto B., Olaho W.M. \& Njogu A.R. Use of tsetse fly data as a measure of trypanosome risk. The Kenya Veterinarian, 1982, 6, 4.

POPPER K. Conjectures et réfutations, la croissance du savoir scientifique. Payot, Paris, 1985.

Rogers D.J. Tsetse density and behaviour as factors in the transmission of trypanosomes. Transactions of the Royal Society of Tropical Medicine and Hygiene, 1979, 73, 131132.

Rogers D.J. Trypanosomiasis "risk" or "challenge". A review with recommendations. Consultation FAO Nairobi, Kenya 13-22 decembre 1979. FAO AGA:TRYP/80/Rep.4, 1980.

Rogers D.J. Trypanosomiasis "risk" or "challenge": a review. Acta tropica, 1985, 42, 5-23.

Rogers D.J. The dynamics of vector-transmited diseases in human communities. Philosophical Transaction of the Royal Society of London B, 1988a, 321, 513-539.

Rogers D.J. A general model for the African trypanosomiases. Parasitology, 1988b, 97, 193-212.

Simo G., Nuiokou F., Nkinin S., Mgbédié M., Laveissière C. \& HERDER S. Étude de la prévalence des infections à trypanosomes chez les animaux sauvages du foyer de la maladie du sommeil de Bipindi, Cameroun. Bulletin de Liaison E Documentation de l'OCEAC, 2000, 33, 8-15.

Smith I.M. \& Rennison B.D. Some factors concerned in trypanosome challenge. ISCTR, Bruxelles, 1958, pp. 63-66.

Wilson A.J., Dar F.K. \& Paris J. A study on the transmission of salivarian trypanosomes isolated from wild tsetse flies. Tropical Animal Health and Production. 1972, 4, 14-22.

Wyatt G.B., Boatin B.A. \& WuraPa F.K. Risk factors associated with the acquisition of sleeping sickness in northeast Zambia; a case-control study. Annal of Tropical Medicine and Parasitology, 1985, 79, 385-392.

Reçu le 23 novembre 2004 Accepté le 24 mars 2005 